\title{
Der Zug ist bereits abgefahren ...
}

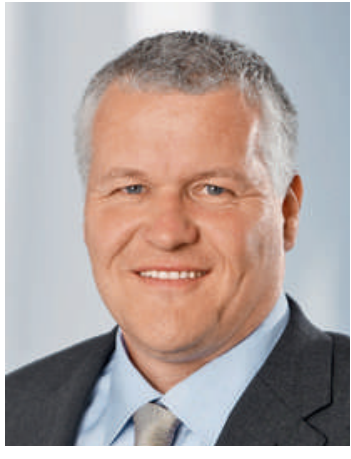

... währenddessen wir immer noch diskutieren, ob wir noch einen Wagon anhängen wollen oder nicht, bleiben wir einfach im Bahnhof stehen. Etwa so kann man die aktuelle gesundheitspolitische Diskussion umschreiben. Zwar ist man sich einig, dass mehr Studienplätze notwendig sind, aber selbst wenn wir diese morgen haben, sind die jungen Kolleginnen und Kollegen nicht vor dem Jahr 2025 zum klinischen Einsatz bzw. in der Grundversorgung bereit. Klar brauchen wir langfristige Konzepte, aber diese sollten nicht die Tagesgeschäfte so absorbieren, dass die kurzund mittelfristige Planung vollständig vergessen geht. Wir benötigen innerhalb der nächsten fünf Jahre einen relevanten Teil der in der Ausbildung stehenden Ärztinnen und Ärzte als Nachwuchs in der Grundversorgung. Diese Planung hätte schon vor zehn Jahren stattfinden sollen, und zwar zu jener Zeit, als der Zulassungsstopp in Kraft war.

\section{Unser Gesundheitswesen benötigt eine überregionale Versorgungsplanung.}

Wie wir ja wissen, kam diesbezüglich vonseiten des Bundes und der Kantone wenig an realisierbaren Projekten, welche national umgesetzt werden könnten. Klar gibt es interessante Projekte wie Gesundheitszentren, welche aber lokal begrenzt sind. Ein Problem bei solchen Planungen ist sicher der typisch schweizerische Föderalismus; dieser ist hauptverantwortlich für die Verhinderung einer überregionalen Versorgungsplanung, welche die freipraktizierende Ärzteschaft ebenso wie die Spitalambulatorien einbezieht. Jeder Kanton hat seine eigene Vorstellung einer Versorgungsplanung, was im Grundsatz nicht falsch ist. Aber es kann nicht sein, dass urbane Regionen aufgrund ihrer Attraktivität den Hauptteil der personellen Fachkraft-Ressourcen absorbieren, während die Peripherie leer ausgeht. Dies hat der letzte Zulassungsstopp klar gezeigt: In Landregionen blieb der Zulassungsstopp Theorie, da der Bedarf an Ärzten sowieso nicht gedeckt werden konnte. Schlimmer noch: Die nachfolgende Generation blieb gezwungenermassen einfach in Warteposition, bis sich eine Möglichkeit einer Praxisübernahme ergab. Dies führte dann zu absurden Situationen im Handel von Praxisbewilligungen.

Aktuell gilt immer noch oft das Prinzip «alles oder nichts», und eine eigentliche Ressourcenplanung (pooling) wird verhindert. Einerseits gibt es Ärztinnen und Ärzte, welche in ihrer Spezialität im Zentrum nicht ausgelastet sind; und andererseits gibt es Regionen, welche genau diese Spezialitäten suchen, jedoch nicht zu 100\%. Wie können wir also diese zwei Situationen sinnvoll nutzen? Unbestritten ist, dass Ärztinnen und Ärzte der fehlenden Attraktivität der Peripherie sich eher für eine Berufstätigkeit in urbanen Regionen entscheiden. Somit müssen Anreize geschaffen werden, welche

\section{Steuern statt stoppen - und dies gemeinsam mit allen Gesundheits-}

\section{partnern.}

diesem Umstand entgegenwirken. Entsprechende Ansätze gibt es bereits, wie z.B. Gesundheitszentren, welche die Infrastruktur zur Verfügung stellen. Dies geschieht hauptsächlich auf Stufe von Gesundheitsfachleuten, Gemeinden und vereinzelt im Rahmen kantonaler Projekte. Aber dringend notwendig wäre eine überregionale Versorgungplanung mit Projekten, welche die Infrastruktur, die Vernetzung, den Informationsaustausch, Qualitätsstandards und eben die Ressourcenplanung einbeziehen.

Die Zeit drängt - und die Akteure sind aufgefordert, kurzund mittelfristige Lösungen zu finden. Der drohende $\mathrm{Zu}$ lassungsstopp verhindert, dass junge, gutausgebildete Ärztinnen und Ärzte an ihrem dringend benötigten Einsatz in der medizinischen Versorgung eingesetzt werden können auf der Basis einer Bedarfsplanung. Es gibt immer noch Kantone, in denen die gesetzlichen Grundlagen für eine zeitgemässe Gesundheitsversorgung nicht vorhanden sind. Auch hier wieder wären gleich lange Spiesse für alle anzustreben. Was hindert einen Kanton, zeitgemässe Rechtsformen für Praxen einzuführen, wenn dies anderswo bestens funktioniert? Das Zauberwort heisst nicht Zulassungsstopp, sondern Zulassungssteuerung mit Förderung von Anreizen, und zwar nur in Zusammenarbeit mit allen Akteuren!

Dr. med. Remo Osterwalder, Mitglied des Zentralvorstandes der FMH, Verantwortlicher Ressort Freipraktizierende Ärztinnen und Ärzte 